\title{
Editorial
}

\section{Artificial Intelligence in Medicine: Passing Hype or the Holy Grail of Solutions?}

\author{
Dirk Wilhelma Nicolas Padoy ${ }^{b}$ \\ a Technical University of Munich, Faculty of Medicine, Klinikum rechts der Isar, Department of

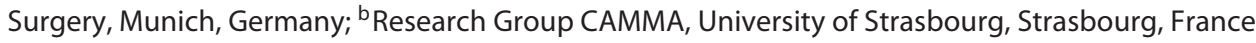

\section{Keywords \\ Artificial intelligence $\cdot$ Smart technology · Transformation · Digitalization}

A "polyhistor" is a person of universal knowledge. In ancient times, there were several such scholars, e.g., Imhotep, Aristoteles, and Albertus Magnus. Gottfried Wilhelm Leibniz, a German scientist and mathematician who died in 1716, was supposedly the last of his kind. Due to the development of science and research since then, being an omniscient expert has become an impossible task. In 1937, in his book World Brain, H.G. Wells proposed "There is no practical obstacle whatsoever now to the creation of an efficient index to all human knowledge, ideas and achievements, to the creation, that is, of a complete planetary memory for all" [1]. He had the vision of a universal library of all sciences which would be augmented each century, according to the increase in knowledge, a vision which has become impossible even with the current available technologies and the Internet. According to the STM report of 2015, the number of published articles grows steadily, by $3-3.5 \% / y e a r$, as does the number of scientific journals, and the number of scientists [2]. The report claimed that 2.5 million articles were published in 2014 alone.

Knowledge is increasing considerably, which also makes it impossible nowadays to become a complete expert, even in a single field. Specializing in a certain field was a temporary solution but has been outpaced by the gain of knowledge. For instance, in recent decades, the field of general surgery was subdivided into the depart- ments of visceral surgery, trauma surgery, vascular surgery, etc. The sub-specialization of visceral surgery led to the fields of upper and lower gastrointestinal surgery, hepato-pancreato-biliary (HPB) surgery, and endocrine surgery, and a further differentiation was made between robotic, minor access, and conventional surgeons. Simultaneously, the elements of a discipline were separated to form new fields, as happened with flexible endoscopy which has emerged as interventional endoscopy.

Despite focusing on only part of the part, being familiar with the content of studies and relevant publications about diseases has truly become a challenge, so that, again, we are losing ground. If we only focus on the details and defined areas of a specialty, however, we will become system-blind and lose the big picture, and thereby fail to understand the complexity of a disease and its broader impact on the human body. To stay competitive, even in the near future, we need powerful support and technologies to provide us with the knowledge necessary to treat our patients.

The term "artificial intelligence (AI)" was coined in 1956 during a workshop on thinking machines; Minsky [3], who first developed artificial neuronal networks, is the supposed founder of AI research. AI comprises the theory and development of computer systems able to perform tasks normally requiring human intelligence, and could likewise be seen as “a system's ability to correctly interpret external data, to learn from such data, and to use those learnings to achieve specific goals and tasks through flexible adaptation" [4]. 
Put simply, AI aims at reconstructing the human brain architecture and processing principles with a computer, but it does not have the same type of intelligence as humans. Human intelligence combines intellect with reason, i.e., the synopsis of individual thoughts; AI is more data-directed and without intuition [5]. However, as constructed on multithreading central processing units (CPUs) and elaborated algorithms, $\mathrm{AI}$ is able to compute a plethora of data in an instant, and, according to Moore's Law, will double its performance within a regular time range [6].

AI therefore has the capacity to analyze the growing number of publications and the increasing amount of knowledge, a feature that we should use to support the daily care and treatment of patients. However, there has been a substantial growth of data, not only in the world of science but also in our clinics in the course of diagnostics and therapy. In 2007, 1 terabyte of data was recorded over the course of a month by the hospitals of a major city; the same amount of data will now be recorded in a single day [7]. With the fostered digitalization of health care processes, the amount of data will rise exponentially. We will need intelligent systems to keep track of this "big data" as well as algorithms to sort, structure, and analyze all the inherent information. AI provides the tools and functionalities required for big data management and has also proved effective in clinical applications.

Examples appear in this edition of Visceral Medicine, in the paper by Jell et al. [8] on the analysis of long-term manometry, and that by Varghese [9] on the analysis of electronic health records. Decision-making support systems (e.g., in oncology) are highlighted as another application [10]. Risk stratification, e.g., concerning postoperative complications [11] or cases of sepsis [12], already works perfectly by means of AI. These examples show how patients' safety could be remarkably improved in the future, but also demonstrate the need for the broad and open-minded provision of clinical data for subsequent analysis. The more data available, the better the trained algorithm will be and can thus, in turn, truly support our clinical work, as has been delineated for computer-based lesion detection in colonoscopy by Wittenberg and Raithel [13]. AI-augmented methods will prove themselves to be a selection criterion, and those who offer them will outperform others.

As a consequence, anyone is invited to provide the floor for AI and support its integration by the adaption of the environment and processes. Medicine, the field of surgery in particular, follows very traditional ways, which do not always offer the required prerequisites for digitalization and process automation. Analogous structures and proprietary solutions are shaping the health care environment and preventing the effective integration of smart technologies. In fact, medicine is only partially ma- chine-readable. If we struggle to evolve our field and open it to the integration of AI methods, i.e., if we want to successfully integrate smart technologies into medicine, there are 2 aspects that become relevant, standardization and interoperability.

Individuality is the figurehead of good and patientcentered medicine, but when AI comes into play, we need reproducible tasks and recurrent patterns to be recognized. Standards are needed. Standardization does not necessarily mean the uniform treatment of patients, but rather the adherence to general principles and the use of established classifications, medical ontologies, and concerted processes. The commonly used classification systems like the APACHE score for sepsis [14], the CHICAGO classification for esophageal motility [8], and the PARIS classification for gastrointestinal adenomas [13] all served as primers for the establishment of artificial neural networks in these fields. Likewise, the standardization of surgical processes, even at a very low level as proposed for cholecystectomy by Immenroth et al. [15], is necessary for the computer-based interpretation of operative interventions and workflow recognition. The articles by Bodenstedt et al. [16] and Chadebecq et al. [17] in this edition of Visceral Medicine explain this in more detail and give a profound insight into its context.

Interoperability can be seen as the second primer for AI-augmented medicine. This relates to the formats and interfaces used for data exchange and transfer. Not without reason, great success has already been achieved in radiology by AI methods. The internationally accepted DICOM standard enables the communication of data between clinicians, researchers, and scientists. Projects like the German OR.NET [18], the Japanese SCOT [19], and the MDPnP in the USA have been initiated to establish comparable protocols for device interoperability in the intervention suite (these appear in the IEEE 11073 and the ORiN standard), and allow for vendor-independent data communication in the medical environment. Likewise, the HL7 FHIR (Fast Healthcare Interoperable Resources) standard was developed to enable a transsectoral data communication of software systems in the health care sector.

These protocols help to merge and compare the data from different sources, which will increase the ground truth and quality of such data, thus enabling the application of AI methods. With the implementation of smart algorithms, interventions and surgical procedures will become safer and more effective. With the interpretation of huge amounts of procedure data as a base, we will be able to identify specific tasks for optimization. Training of young physicians could also be brought to a new level, if AI evaluates and rates their performance during interventions and compares them to that of an expert. The article by Brown et al. [20] points in this direction and 
explains how skills assessment could work in an AI environment.

Although the concept of AI has been around for almost 60 years, it is still in its infancy, at least in medicine. Current applications of AI are labeled as weak as they only perform well in narrow and defined fields; strong AI, which will perform in a more human-like manner and on a more general basis, is not to be expected before $2040 \mathrm{ac}$ cording to an assessment by the experts [21]. We need AI in medicine to cope with the challenges of big data and the exponential increase of knowledge, but also with the bureaucratization and concentration of work. To facilitate the successful implementation of AI in health care, we are asked to reshape our principles and our mindset. Without doubt, this might be one of the greatest tasks the medical fraternity has faced in recent years, but it offers the chance for us to dedicate ourselves to our patients once again and provide them with an unrivaled quality of care.

Those still critical about the application of AI in health care must remember the commitment each one of us made to bring the best to our patients. If AI-supported methods are proven effective and superior to the conventional approach, it is not up to us to decide whether or not to apply the technology. Ethically, we will be compelled to use it.

\section{Conflict of Interest Statement}

D.W. and N.P. have no conflicts of interest to declare.

\section{Funding Sources}

There was no funding.

\section{Author Contributions}

Both authors contributed equally to the completion, revision, and finalization of this editorial.

\section{References}

1 Rayward W. H.G. Wells's Idea of a World Brain: A Critical Reassessment. JASIS. 1999; 50(7):557-73.

2 Ware M, Mabe M: The STM report: An overview of scientific and scholarly journal publishing, 4th ed. The Hague: STM; 2015.

3 Minsky M. Steps toward artificial intelligence. Proc IRE. 1961;49(1):8-30.

4 Kaplan A, Haenlein M. Siri, Siri, in my hand: who's the fairest in the land? On the interpretations, illustrations, and implications of artificial intelligence. Bus Horiz. 2019;62(1):1525.

5 Hirsch MC. KI und neue Wege der Care-Navigation. Der Klinikarzt. 2020;49(06):256-61.

6 Schaller RR. Moore's law: past, present and future. IEEE Spectr. 1997;34(6):52-9.

7 Faber O, editor. Digitalisierung - ein Megatrend: Treiber \& Technologische Grundlagen. Management 40 - Unternehmensführung im digitalen Zeitalter. Berlin: Springer; 2019. p. 3-42.

8 Jell A, Kuttler C, Ostler D, Hüser N. How to Cope with Big Data in Functional Analysis of the Esophagus. Visc Med. 2020. DOI: $10.1159 / 000511931$.

9 Varghese J. Artificial Intelligence in Medicine: Chances and Challenges for Wide Clinical Adoption. Visc Med. 2020;36(6):1-7.
10 Klarenbeek SE, Weekenstroo HH, Sedelaar JP, Fütterer JJ, Prokop M, Tummers M. The Effect of Higher Level Computerized Clinical Decision Support Systems on Oncology Care: A Systematic Review. Cancers (Basel). 2020 Apr;12(4):E1032.

11 Merath K, Hyer JM, Mehta R, Farooq A, Bagante F, Sahara K, et al. Use of Machine Learning for Prediction of Patient Risk of Postoperative Complications After Liver, Pancreatic, and Colorectal Surgery. J Gastrointest Surg. 2020 Aug;24(8):1843-51.

12 Fleuren LM, Klausch TL, Zwager CL, Schoonmade LJ, Guo T, Roggeveen LF, et al. Machine learning for the prediction of sepsis: a systematic review and meta-analysis of diagnostic test accuracy. Intensive Care Med. 2020 Mar;46(3):383-400.

13 Wittenberg T, Raithel M. Artificial Intelligence-Based Polyp Detection in Colonoscopy - Where Do We Come from, Where Do We Stand, and Where Will We Go to? Visc Med. 2020. DOI: 10.1159/000512438.

14 Mofidi R, Duff MD, Madhavan KK, Garden OJ, Parks RW. Identification of severe acute pancreatitis using an artificial neural network. Surgery. 2007 Jan;141(1):59-66.
15 Immenroth M, Bürger T, Brenner J, Nagelschmidt M, Eberspächer H, Troidl H. Mental training in surgical education: a randomized controlled trial. Ann Surg. 2007 Mar;245(3): 385-91.

16 Bodenstedt S, Wagner M, Müller-Stich BP, Weitz J, Speidel S. AI-Assisted Surgery - Potential and Challenges. Visc Med. 2020. DOI: $10.1159 / 000511351$.

17 Chadebecq F, Vasconcelos F, Mazomenos E, Stoyanov D. Computer Vision in the Surgical Operating Room. Visc Med. 2020;36(6):1-7.

18 Kasparick M, Schmitz M, Andersen B, Rockstroh M, Franke S, Schlichting S, et al. OR. NET: a service-oriented architecture for safe and dynamic medical device interoperability. Biomed Tech (Berl). 2018 Feb;63(1):11-30.

19 Okamoto J, Masamune K, Iseki H, Muragaki Y. Development concepts of a Smart Cyber Operating Theater (SCOT) using ORiN technology. Biomed Tech (Berl). 2018 Feb;63(1):31-7.

20 Brown K, Bhattacharyya K, Kulason S, Aneq Zia A, Jarca A. How to Bring Surgery to the Next Level: Intepretable Skills Assessment in Robotic-Assisted Surgery. Visc Med. 2020. DOI: $10.1159 / 000512437$.

21 Müller VC, Bostrom N. Future progress in artificial intelligence: A survey of expert opinion. In: Müller VC, editor. Fundamental Issues of Artificial Intelligence. Springer; 2016. p. $555-72$. 